\title{
Global Solution of a Nonlinear Conservation Law with Weak Discontinuous Flux in the Half Space
}

\author{
Xiaoqian Li ${ }^{*}$, Jing Zhang² \\ ${ }^{1}$ Department of Mathematics, Jinan University, Guangzhou, China \\ ${ }^{2}$ Department of Optoelectronic Engineering, Jinan University, Guangzhou, China \\ Email: ${ }^{*} 15813341397 @ 163 . c o m$
}

How to cite this paper: $\mathrm{Li}, \mathrm{X} . \mathrm{Q}$. and Zhang, J. (2018) Global Solution of a Nonlinear Conservation Law with Weak Discontinuous Flux in the Half Space. American Journal of Computational Mathematics, 8, 326-342.

https://doi.org/10.4236/ajcm.2018.84026

Received: December 12, 2018

Accepted: December 26, 2018

Published: December 29, 2018

Copyright (c) 2018 by authors and Scientific Research Publishing Inc. This work is licensed under the Creative Commons Attribution International License (CC BY 4.0).

http://creativecommons.org/licenses/by/4.0/

(c) (i) Open Access

\section{Abstract}

This paper is concerned with the initial-boundary value problem of a nonlinear conservation law in the half space $R^{+}=\{x \mid x>0\}$

$$
\left\{\begin{array}{l}
u_{t}+f(u)_{x}=0, x \in R^{+}, t>0 \\
u(x, 0)= \begin{cases}u_{m}, & 0<x<a \\
u_{+}, & x>a\end{cases} \\
u(0, t)=u_{-}, t>0
\end{array}\right.
$$

where $a>0, u(x, t)$ is an unknown function of $x \in R^{+}$and $t>0, u_{ \pm}, u_{m}$ are three given constants satisfying $u_{m}=u_{+} \neq u_{-}$or $u_{m}=u_{-} \neq u_{+}$, and the flux function $f$ is a given continuous function with a weak discontinuous point $u_{d}$. The main purpose of our present manuscript is devoted to studying the structure of the global weak entropy solution for the above initial-boundary value problem under the condition of $f_{-}^{\prime}\left(u_{d}\right)>f_{+}^{\prime}\left(u_{d}\right)$. By the characteristic method and the truncation method, we construct the global weak entropy solution of this initial-boundary value problem, and investigate the interaction of elementary waves with the boundary and the boundary behavior of the weak entropy solution.

\section{Keywords}

Nonlinear Conservation Laws with Weak Discontinuous Flux, Initial-Boundary Value Problem, Shock Wave, Rarefaction Wave, Contact Discontinuity, Interaction, Structure of Global Weak Entropy Solution

\section{Introduction}

Consider the initial-boundary value problem of a nonlinear conservation law in 
the half space $R^{+}=\{x \mid x>0\}$

$$
u_{t}+f(u)_{x}=0, x \in R^{+}, t>0
$$

with the initial condition

$$
u(x, 0)=u_{0}(x):= \begin{cases}u_{m}, & 0<x<a \\ u_{+}, & x>a\end{cases}
$$

and the boundary condition

$$
u(0, t)=u_{b}(t):=u_{-}, t>0
$$

where $a>0, u_{ \pm}$and $u_{m}$ are constant, and the flux $f$ is a given continuous function of $u$, which satisfies the following conditions:

$\left(\mathrm{A}_{1}\right)$ Its derivative function $f^{\prime}$ is piecewise $C$-smooth with one discontinuous point $u_{d}$, and $f_{ \pm}^{\prime}\left(u_{d}\right)$ exists, where $f_{-}^{\prime}$ and $f_{+}^{\prime}$ represent the left and right derivatives of $f$, respectively;

$\left(\mathrm{A}_{2}\right) f^{\prime \prime}(u)>0$ for $u \neq u_{d}$.

For such an initial-boundary value problem (2)-(4), under the conditions of $\left(A_{1}\right)$ and $\left(A_{2}\right)$, the global weak entropy solution was constructed in [1] for the case of $f_{-}^{\prime}\left(u_{d}\right)<f_{+}^{\prime}\left(u_{d}\right)$. We want to study the structure of the global weak entropy solution of the problem (2)-(4) for the case of $f_{-}^{\prime}\left(u_{d}\right)>f_{+}^{\prime}\left(u_{d}\right)$ under the conditions of $\left(\mathrm{A}_{1}\right)$ and $\left(\mathrm{A}_{2}\right)$. As first step, we investigate the Riemann type of initial-boundary value problem, i.e., the problem (2)-(4) with $u_{m}=u_{+} \neq u_{-}$or $u_{m}=u_{-} \neq u_{+}$in our present manuscript. The more general problem (2)-(4) with $u_{m} \neq u_{ \pm}$will be investigated in our forthcoming paper.

The main difficulty in studying the initial-boundary value problem of hyperbolic conservation laws is that the appearance of boundary results in obstacle in analysis. The difficulty lies in two respects: on one hand, the initial-boundary value problem of hyperbolic conservation laws is generally ill-posed; on the other hand, the nonlinear elementary waves will perhaps collide and interact with the boundary at finite time, so that the boundary layer may appear, which requires to give a reasonable boundary entropy condition to ensure the well-posedness of the global weak solution satisfying the relevant physical meaning. Bardos-Leroux-Nedelec [2] first established the existence and uniqueness of global weak entropy solution in the BV-setting for the initial-boundary value problem of scalar conservation laws with several space variables by vanishing viscosity method and by Kruzkov's method, respectively, and they gave a boundary entropy condition which requires only that the boundary data and the boundary value of solution satisfy an inequality. The other results of existence and uniqueness have been done for the initial-boundary value problem of scalar conservation laws after [2]. The interested readers are referred to [3]-[9]. Because of the influence of boundary, the geometric structure of the solution of initial-boundary value problem for scalar conservation laws is much more difficult than that of corresponding Cauchy problem. In recent years, for the initial-boundary value problem of one-dimensional nonlinear hyperbolic conservation laws (2)-(4) with $\mathrm{C}^{2}$-smooth flux, some results have been obtained in this 
regard. The authors in papers [10] [11] [12] constructed the global weak entropy solutions to the initial-boundary problems on a bounded interval for some special initial-boundary data with three pieces of constant corresponding to the practical problem of continuous sedimentation of an ideal suspension. Liu-Pan [13] [14] [15] gave a construction method to the global weak entropy solution of the initial-boundary value problem with piecewise smooth initial dada and constant boundary data for scalar nonlinear hyperbolic conservation laws, and clarified the structure and boundary behavior of the weak entropy solution.

The present paper is organized as follows. In Section 2, we introduce the definition of weak entropy solution and the boundary entropy condition for the initial-boundary value problem (2)-(4) and give a lemma to be used to construct the piecewise smooth solution of (2)-(4). In Section 3, based on the analysis method in [13], we use the lemma on piecewise smooth solution given in Section 2 to construct the global weak entropy solution of the initial-boundary value problem (2)-(4) with $u_{m}=u_{+} \neq u_{-}$or $u_{m}=u_{-} \neq u_{+}$under the conditions of $\left(\mathrm{A}_{1}\right)$ and $\left(\mathrm{A}_{2}\right)$ for the case of $f_{-}^{\prime}\left(u_{d}\right)>f_{+}^{\prime}\left(u_{d}\right)$, and state the geometric structure and the behavior of boundary for the weak entropy solution.

\section{Definition of Weak Entropy Solution and Related Lemma}

Following the papers [2] [3], we give the definition and the boundary entropy condition of weak entropy solution for the initial- boundary value problem (2)-(4).

Definition 1 Let $u(x, t)$ be a bounded and local bounded variation function on $[0, \infty) \times[0, \infty)$. If for each $k \in(-\infty, \infty)$ and for any nonnegative test function $\phi \in C_{0}^{\infty}([0, \infty) \times[0, \infty))$, it satisfies the following inequality

$$
\begin{aligned}
& \int_{0}^{\infty} \int_{0}^{\infty}\left(|u-k| \phi_{t}+\operatorname{sgn}(u-k)(f(u)-f(k)) \phi_{x}\right) \mathrm{d} x \mathrm{~d} t \\
& +\int_{0}^{\infty}\left|u_{0}(x)-k\right| \phi(x, 0) \mathrm{d} x \\
& +\int_{0}^{\infty} \operatorname{sgn}\left(u_{b}(t)-k\right)(f(u(0, t))-f(k)) \phi(0, t) \mathrm{d} t \geq 0,
\end{aligned}
$$

where

$$
\operatorname{sgn}(x)= \begin{cases}1 & x \geq 0 \\ -1 & x<0\end{cases}
$$

then $u(x, t)$ is called a weak entropy solution of the initial-boundary problem (2)-(4).

Lemma 1 If $u(x, t)$ is a weak entropy solution of (2)-(4), then it satisfies the following boundary entropy condition:

$$
\begin{gathered}
u(0+, t)=u_{b}(t) \\
\text { or } \frac{f(u(0+, t))-f(k)}{u(0+, t)-k} \leq 0, k \in I\left(u(0+, t), u_{b}(t)\right), k \neq u(0+, t) \text {, a.e. } t \geq 0,
\end{gathered}
$$

where $k \in I\left(u(0+, t), u_{b}(t)\right)=\left[\min \left\{u(0+, t), u_{b}(t)\right\}, \max \left\{u(0+, t), u_{b}(t)\right\}\right]$.

For the initial-boundary value problem (2)-(4) with general initial-boundary 
data of bounded variation, its global weak entropy solution in the sense of (5) exists and is unique (see [2] [3] [6] [11]). In order to clarify the structure of the global weak entropy solution for the initial-boundary value problem (2)-(4) under the assumptions $\left(A_{1}\right)$ and $\left(A_{2}\right)$, we need the following lemma 2 .

Lemma 2 Suppose that the conditions $\left(A_{1}\right)$ and $\left(A_{2}\right)$ are valid. A piecewise smooth function $u(x, t)$ with piecewise smooth discontinuity curves is a weak entropy solution of (2)-(4) in the sense of (5) if and only if the following conditions are satisfied:

1) $u(x, t)$ satisfies the Equation (2) on its smooth domains;

2) If $x=x(t)$ is a weak discontinuity curves of $u(x, t)$, then when $u(x(t), t)$ is not the discontinuous point of $f^{\prime}, \frac{\mathrm{d} x}{\mathrm{~d} t}=f^{\prime}(u(x(t), t))$, and when $u(x(t), t)$ is the discontinuous point of $f^{\prime}$,

$$
\frac{\mathrm{d} x}{\mathrm{~d} t}=f_{-}^{\prime}(u(x(t), t)) \text { or } \frac{\mathrm{d} x}{\mathrm{~d} t}=f_{+}^{\prime}(u(x(t), t)) ;
$$

If $x=x(t)$ is a strong discontinuity curves of $u(x, t)$, then the Rankine-Hugoniot's discontinuity condition

$$
\frac{\mathrm{d} x}{\mathrm{~d} t}=\frac{f\left(u_{-}\right)-f\left(u_{+}\right)}{u_{-}-u_{+}}
$$

and the Oleinik's entropy condition

$$
\frac{f\left(u_{-}\right)-f\left(u_{+}\right)}{u_{-}-u_{+}} \leq \frac{f\left(u_{-}\right)-f(u)}{u_{-}-u}
$$

hold, where $u_{ \pm}=u(x(t) \pm 0, t)$, and $u$ is any number between $u_{-}$and $u_{+}$.

3) The boundary entropy condition (6) is valid.

4) $u(x, 0)=u_{0}(x)$ a.e. $x \geq 0$.

By using the analogous technique in references [3] [16], Lemma 2 is easy to be proved by Definition 1 and Lemma 1, we omit it here.

\section{Solution Structures}

In this section, for the initial-boundary value problem (2)-(4) with $u_{m}=u_{+} \neq u_{-}$ or $u_{m}=u_{-} \neq u_{+}$, we shall construct the global weak entropy solution under the conditions of $(\mathrm{A})_{1}$ and $(\mathrm{A})_{2}$ and $f_{-}^{\prime}\left(u_{d}\right)>f_{+}^{\prime}\left(u_{d}\right)$ by employing Lemma 2 and the structure of weak entropy solution of the corresponding initial value problem, and investigate the interaction of elementary waves with the boundary $x=0$ and the boundary behaviors of the global weak entropy solution. The methods which will be used to construct the weak entropy solutions of the initial value problem and the initial-boundary value problem here are the characteristic method (see also [17]) and the truncation method developed in [13], respectively. We only discuss the case of $f_{-}^{\prime}\left(u_{d}\right)>0>f_{+}^{\prime}\left(u_{d}\right)$. The other cases can be dealt with similarly.

For the convenience of our construction work, we first introduce some notations. We denote 


$$
\omega\left(u_{1}, u_{2}\right)=\frac{f\left(u_{1}\right)-f\left(u_{2}\right)}{u_{1}-u_{2}}
$$

Let $R\left(u_{l}, u_{r} ;(b, c)\right)$ denote a rarefaction wave connecting $u_{l}$ and $u_{r}$ from the left to the right, centered at point $(b, c)$ in the $x-t$ plane, and $S\left(u_{l}, u_{r} ;(b, c)\right)$ denote a shock wave $x=x(t)$ connecting $u_{l}$ and $u_{r}$ from the left to the right, starting at point $(b, c)$ in the $x-t$ plane, where $x=x(t)$ satisfies (7), (8) and the Lax's shock wave condition $f_{-}^{\prime}\left(u_{-}\right)>x^{\prime}(t)>f_{+}^{\prime}\left(u_{+}\right)$. We denote a left or right or double-contact discontinuity wave $x=x(t)$ connecting $u_{l}$ and $u_{r}$ from the left to the right, emanating from point $(b, c)$ in the $x-t$ plane by $S^{l}\left(u_{l}, u_{r} ;(b, c)\right)$ or $S^{r}\left(u_{l}, u_{r} ;(b, c)\right)$ or $S^{l r}\left(u_{l}, u_{r} ;(b, c)\right)$, respectively, where $x=x(t)$ satisfies (7), (8) and the contact condition $f_{-}^{\prime}\left(u_{-}\right)=x^{\prime}(t)>f_{+}^{\prime}\left(u_{+}\right)$or $f_{-}^{\prime}\left(u_{-}\right)>x^{\prime}(t)=f_{+}^{\prime}\left(u_{+}\right)$or $f_{-}^{\prime}\left(u_{-}\right)=x^{\prime}(t)=f_{+}^{\prime}\left(u_{+}\right)$, respectively. The left or right or double-contact discontinuity waves are collectively referred to as the contact- discontinuity waves. It is well known that the solution of the shock wave $S\left(u_{l}, u_{r} ;(b, c)\right.$ ) (or the contact-discontinuity waves $S^{l}\left(u_{l}, u_{r} ;(b, c)\right)$ or $S^{r}\left(u_{l}, u_{r} ;(b, c)\right)$ or $S^{l r}\left(u_{l}, u_{r} ;(b, c)\right)$ and the solution of the central rarefaction wave $R\left(u_{l}, u_{r} ;(b, c)\right)$ in $x-t$ plane is respectively expressed as:

$$
u(x, t)= \begin{cases}u_{l}, & x<b+\omega\left(u_{l}, u_{r}\right)(t-c) \\ u_{r}, & x>b+\omega\left(u_{l}, u_{r}\right)(t-c)\end{cases}
$$

and

$$
u(x, t)= \begin{cases}u_{l}, & x<b+f_{+}^{\prime}\left(u_{l}\right)(t-c) \\ \left(f^{\prime}\right)^{-1}\left(\frac{x-b}{t-c}\right), & b+f_{+}^{\prime}\left(u_{l}\right)(t-c)<x<b+f_{-}^{\prime}\left(u_{r}\right)(t-c) \\ u_{+}, & x>b+f_{-}^{\prime}\left(u_{r}\right)(t-c)\end{cases}
$$

where $t>c$.

If $v(x, t)$ is an increasing (or decreasing) function with respect to $x$, which connects $u_{-}$and $u_{+}$from the leftmost to the rightmost, then $v(x, t)$ is called an expansion wave (or compression wave) connecting $u_{-}$and $u_{+}$, we denote it by $E\left(u_{-}, u_{+}\right)$(or $\left.C\left(u_{-}, u_{+}\right)\right)$.

When $u_{ \pm} \leq u_{d}$ or $u_{ \pm} \geq u_{d}$, the problem (2)-(4) is degenerated into a corresponding problem with $f \in C^{2}$ (see [13]). Throughout this section, we always suppose that $\left(u_{-}-u_{d}\right)\left(u_{+}-u_{d}\right)<0$. We divide our problem into two cases: (I) $u_{m}=u_{+} \neq u_{-}$; (II) $u_{m}=u_{-} \neq u_{+}$.

\subsection{Case (I): $u_{m}=u_{+} \neq u_{-}$}

According to the discussion framework in [13], we first investigate the solution structure of the following Riemann problem

$$
\left\{\begin{array}{l}
v_{t}+f(v)_{x}=0,-\infty<x<\infty, t>0 \\
v(x, 0)= \begin{cases}u_{-}, & x<0 \\
u_{+}, & x>0\end{cases}
\end{array}\right.
$$


and then by which and Lemma 2, we construct the global weak entropy solution for the initial-boundary problem (2)-(4).

We divide this case into two sub-cases: 1) $u_{+}<u_{d}<u_{-}$;2) $u_{-}<u_{d}<u_{+}$.

3.1.1. $u_{+}<u_{d}<u_{-}$

Let $u_{1^{*}}$ denote the abscissa of the intersection point of the secant passing through point $\left(u_{-}, f\left(u_{-}\right)\right)$and $\left(u_{d}, f\left(u_{d}\right)\right)$ with the image of $f$ in $u-f(u)$ plane (see Figure 1).

If $u_{+} \leq u_{1^{*}}$, similar to the discussion in [17], the weak entropy solution $v(x, t)$ of Riemann problem (12) includes only a shock wave $S\left(u_{-}, u_{+} ;(0,0)\right)$ starting at point $(0,0)$ (see Figure 2$)$, and this shock wave solution can be expressed as follows:

$$
v(x, t)= \begin{cases}u_{-}, & x<\omega\left(u_{-}, u_{+}\right) t \\ u_{+}, & x>\omega\left(u_{-}, u_{+}\right) t\end{cases}
$$

Let $u(x, t)=\left.v(x, t)\right|_{x, t>0}$, then

$$
u(0+,) t=\left\{\begin{array}{l}
u_{+}, \text {as } \omega\left(u_{-}, u_{+}\right) \leq 0 \\
u_{-}, \text {as } \omega\left(u_{-}, u_{+}\right)>0
\end{array}\right.
$$

We can easily verify that this $u(x, t)$ satisfies all conditions in Lemma 2, therefore it is the global weak entropy solution of the initial-boundary problem (2)-(4). $u(x, t)$ includes only a constant state $u_{+}$as $\omega\left(u_{-}, u_{+}\right) \leq 0$ or a shock wave $S\left(u_{-}, u_{+} ;(0,0)\right)$ as $\omega\left(u_{-}, u_{+}\right)>0$ (see Figure 2).

If $u_{1^{*}}<u_{+}<u_{d}$, by $\omega\left(u_{-}, u_{d}\right)<\omega\left(u_{d}, u_{+}\right),(7)$, (8) and the Lax's shock wave condition, we have that a compression wave $C\left(u_{-}, u_{+}\right)$, which includes two shock waves $S\left(u_{-}, u_{d} ;(0,0)\right)$ and $S\left(u_{d}, u_{+} ;(0,0)\right)$, i.e., $C\left(u_{-}, u_{+}\right)=S\left(u_{-}, u_{d} ;(0,0)\right) \cup S\left(u_{d}, u_{+} ;(0,0)\right)$, appears in the weak entropy solution $v(x, t)$ of the Riemann problem (12) (see Figure 3), this compression wave solution $v(x, t)$ can be written as:

$$
v(x, t)= \begin{cases}u_{-}, & x<\omega\left(u_{-}, u_{d}\right) t \\ u_{d}, & \omega\left(u_{-}, u_{d}\right) t<x<\omega\left(u_{d}, u_{+}\right) t \\ u_{+}, & x>\omega\left(u_{d}, u_{+}\right) t .\end{cases}
$$

Set $u(x, t)=\left.v(x, t)\right|_{x, t>0}$, then it holds

$$
u(0+, t)= \begin{cases}u_{-}, & \text {as } \omega\left(u_{-}, u_{d}\right)>0 \\ u_{d}, & \text { as } \omega\left(u_{-}, u_{d}\right) \leq 0 \text { and } \omega\left(u_{d}, u_{+}\right)>0 \\ u_{+}, & \text {as } \omega\left(u_{d}, u_{+}\right) \leq 0\end{cases}
$$

By Lemma 2, this $u(x, t)$ is the global weak entropy solution of the initial-boundary problem (2)-(4). $u(x, t)$ includes only a compression wave $C\left(u_{-}, u_{+}\right)=S\left(u_{-}, u_{d} ;(0,0)\right) \cup S\left(u_{d}, u_{+} ;(0,0)\right)$ as $\omega\left(u_{-}, u_{d}\right)>0$ or a shock wave $S\left(u_{d}, u_{+} ;(0,0)\right)$ as $\omega\left(u_{-}, u_{d}\right) \leq 0$ and $\omega\left(u_{d}, u_{+}\right)>0$ or a constant state $u_{+}$as $\omega\left(u_{d}, u_{+}\right) \leq 0$ (see Figure 3 ). 


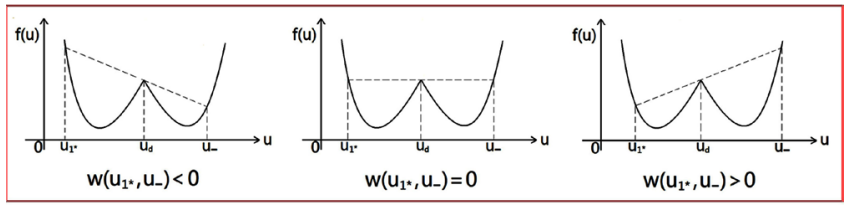

Figure 1. The location of $u_{1^{*}}$ in the $u-f(u)$ plane.

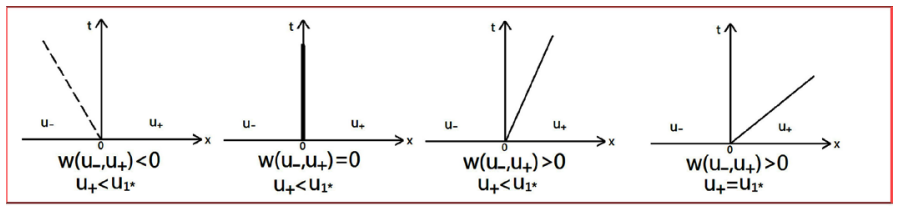

Figure 2. The shock wave $S\left(u_{-}, u_{+}\right)$of the problem (12) for the case of $u_{+} \leq u_{1^{*}}$.

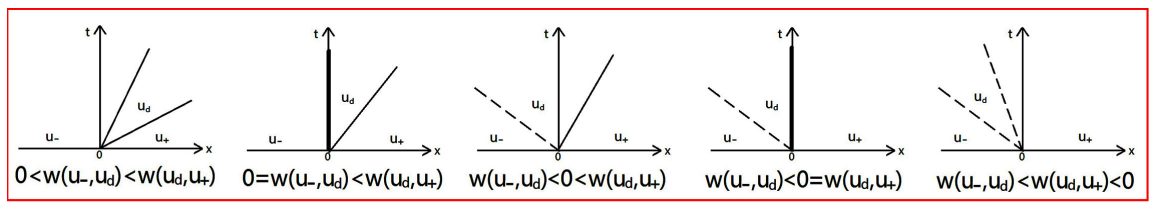

Figure 3. The compression wave $C\left(u_{-}, u_{+}\right)$of the problem (12) for the case of $u_{1^{*}}<u_{+}<u_{d}$.

\subsection{2. $u_{-}<u_{d}<u_{+}$}

By the assumptions on the flux function $f$, there exist two numbers $u_{2^{*}}, u_{3^{*}}$ such that

$u_{2^{*}}<u_{d}<u_{3^{*}}$ and $\omega\left(u_{2^{*}}, u_{3^{*}}\right)=f^{\prime}\left(u_{2}\right)=f^{\prime}\left(u_{3}\right)$ (see Figure 4). By using of the similar analysis in [17], the weak entropy solution $v(x, t)$ of Riemann problem (12) includes only an expansion wave $E\left(u_{-}, u_{+}\right)$.

When $u_{-}<u_{2^{*}}, E\left(u_{-}, u_{+}\right)$can be expressed as follows:

$$
E\left(u_{-}, u_{+}\right)=\left\{\begin{array}{l}
R\left(u_{-}, u_{2^{*}} ;(0,0)\right) \cup S^{l r}\left(u_{2^{*}}, u_{3^{*}} ;(0,0)\right), \text { if } u_{+}=u_{3^{*}} \\
R\left(u_{-}, u_{2^{*}} ;(0,0)\right) \cup S^{l r}\left(u_{2^{*}}, u_{3^{*}} ;(0,0)\right) \cup R\left(u_{3^{*}}, u_{+} ;(0,0)\right), \text { if } u_{+}>u_{3^{*}} \\
R\left(u_{-}, u_{4^{*}} ;(0,0)\right) \cup S^{l}\left(u_{4^{*}}, u_{+} ;(0,0)\right), \text { if } u_{d}<u_{+}<u_{3^{*}},
\end{array}\right.
$$

where $u_{4^{*}} \in\left(u_{2^{*}}, u_{d}\right)$ satisfies $\omega\left(u_{4^{*}}, u_{+}\right)=f^{\prime}\left(u_{4^{*}}\right)$. Thus this expansion wave solution can be written as:

$$
v(x, t)=\left\{\begin{array}{l}
u_{-}, \quad x \leq f^{\prime}\left(u_{-}\right) t \\
\left(f^{\prime}\right)^{-1}\left(\frac{x}{t}\right), \quad f^{\prime}\left(u_{-}\right) t<x<f^{\prime}\left(u_{2^{*}}\right) t \\
u_{+}, \quad x>f^{\prime}\left(u_{2^{*}}\right) t
\end{array}\right.
$$

for $u_{+}=u_{3^{*}}$ (see Figure 5), and for $u_{+}>u_{3^{*}}$,

$$
v(x, t)=\left\{\begin{array}{l}
u_{-}, \quad x \leq f^{\prime}\left(u_{-}\right) t \\
\left(f^{\prime}\right)^{-1}\left(\frac{x}{t}\right), \quad f^{\prime}\left(u_{-}\right) t<x<f^{\prime}\left(u_{2^{*}}\right) t \text { or } f^{\prime}\left(u_{3^{*}}\right) t<x \leq f^{\prime}\left(u_{+}\right) t \\
u_{+}, \quad x>f^{\prime}\left(u_{+}\right) t
\end{array}\right.
$$



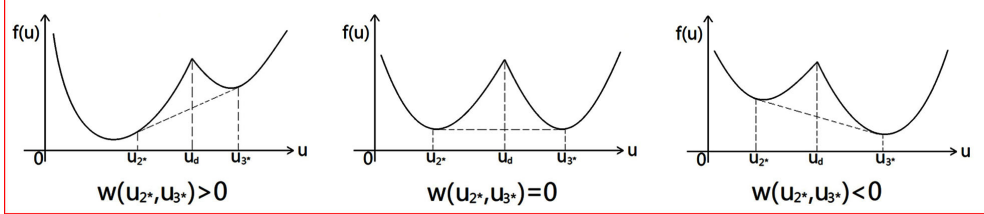

Figure 4. The location of $u_{2^{*}}$ and $u_{3^{*}}$ in the $u-f(u)$ plane.

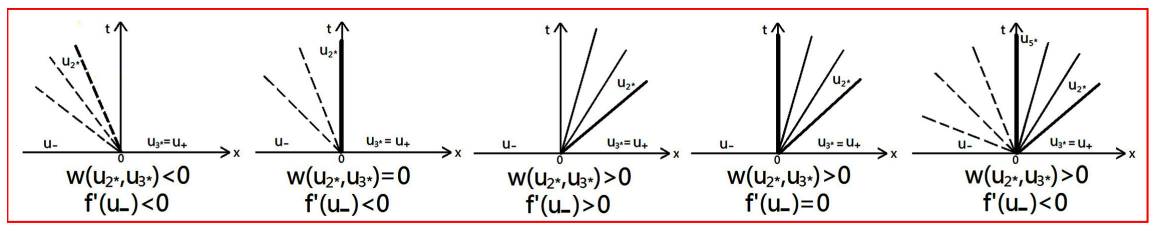

Figure 5. The expansion wave $E\left(u_{-}, u_{+}\right)$of the problem (12) for the case of $u_{+}=u_{3^{*}}$.

(see Figure 6), and for $u_{d}<u_{+}<u_{3^{*}}$,

$$
v(x, t)=\left\{\begin{array}{l}
u_{-}, \quad x \leq f^{\prime}\left(u_{-}\right) t \\
\left(f^{\prime}\right)^{-1}\left(\frac{x}{t}\right), \quad f^{\prime}\left(u_{-}\right) t<x<f^{\prime}\left(u_{4^{*}}\right) t \\
u_{+}, \quad x>f^{\prime}\left(u_{4^{*}}\right) t
\end{array}\right.
$$

(see Figure 7).

If we take $u(x, t)=\left.v(x, t)\right|_{x, t>0}$, then for the case of $u_{+}=u_{3^{*}}$,

$$
u(0+, t)= \begin{cases}u_{+}, & \text {if } \omega\left(u_{2^{*}}, u_{3^{*}}\right) \leq 0 \\ u_{-}, & \text {if } \omega\left(u_{2^{*}}, u_{3^{*}}\right)>0 \text { and } f^{\prime}\left(u_{-}\right) \geq 0 \\ u_{5^{*}}, & \text { if } \omega\left(u_{2^{*}}, u_{3^{*}}\right)>0 \text { and } f^{\prime}\left(u_{-}\right)<0\end{cases}
$$

where $u_{5^{*}} \in\left(u_{-}, u_{2^{*}}\right)$ satisfies $f^{\prime}\left(u_{5^{*}}\right)=0$; and for the case of $u_{+}>u_{3^{*}}$,

$$
u(0+, t)=\left\{\begin{array}{l}
u_{+}, \text {if } f^{\prime}\left(u_{+}\right) \leq 0 \\
u_{6^{*}}, \text { if } f^{\prime}\left(u_{+}\right)>0 \text { and } \omega\left(u_{2^{*}}, u_{3^{*}}\right) \leq 0 \\
u_{-}, \text {if } f^{\prime}\left(u_{+}\right)>0 \text { and } \omega\left(u_{2^{*}}, u_{3^{*}}\right)>0 \text { and } f^{\prime}\left(u_{-}\right) \geq 0 \\
u_{5^{*}}, \text { if } f^{\prime}\left(u_{+}\right)>0 \text { and } \omega\left(u_{2^{*}}, u_{3^{*}}\right)>0 \text { and } f^{\prime}\left(u_{-}\right)<0
\end{array}\right.
$$

where $u_{6^{*}} \in\left[u_{3^{*}}, u_{+}\right)$satisfies $f^{\prime}\left(u_{6^{*}}\right)=0$; and for the case of $u_{d}<u_{+}<u_{3^{*}}$,

$$
u(0+, t)= \begin{cases}u_{+}, & \text {if } \omega\left(u_{4^{*}}, u_{+}\right) \leq 0 \\ u_{-}, & \text {if } \omega\left(u_{4^{*}}, u_{+}\right)>0 \text { and } f^{\prime}\left(u_{-}\right) \geq 0 \\ u_{5^{*}}, & \text { if } \omega\left(u_{4^{*}}, u_{+}\right)>0 \text { and } f^{\prime}\left(u_{-}\right)<0\end{cases}
$$

Therefore, from Lemma 2, we can also easily verify that $u(x, t)$ is the global weak entropy solution of the problem (2)-(4). $u(x, t)$ includes only an expansion wave $\bar{E}=\left.E\left(u_{-}, u_{+}\right)\right|_{x, t>0}$, which does not interact with the boundary $x=0$ and can be written as follows:

$\bar{E}=\left\{\begin{array}{l}u_{+}, \text {if } \omega\left(u_{2^{*}}, u_{3^{*}}\right) \leq 0 \\ R\left(u_{-}, u_{2^{*}} ;(0,0)\right) \cup S^{l r}\left(u_{2^{*}}, u_{3^{*}} ;(0,0)\right), \quad \text { if } \omega\left(u_{2^{*}}, u_{3^{*}}\right)>0 \text { and } f^{\prime}\left(u_{-}\right) \geq 0 \\ R\left(u_{5^{*}}, u_{2^{*}} ;(0,0)\right) \cup S^{l r}\left(u_{2^{*}}, u_{3^{*}} ;(0,0)\right), \text { if } \omega\left(u_{2^{*}}, u_{3^{*}}\right)>0 \text { and } f^{\prime}\left(u_{-}\right)<0\end{array}\right.$ 


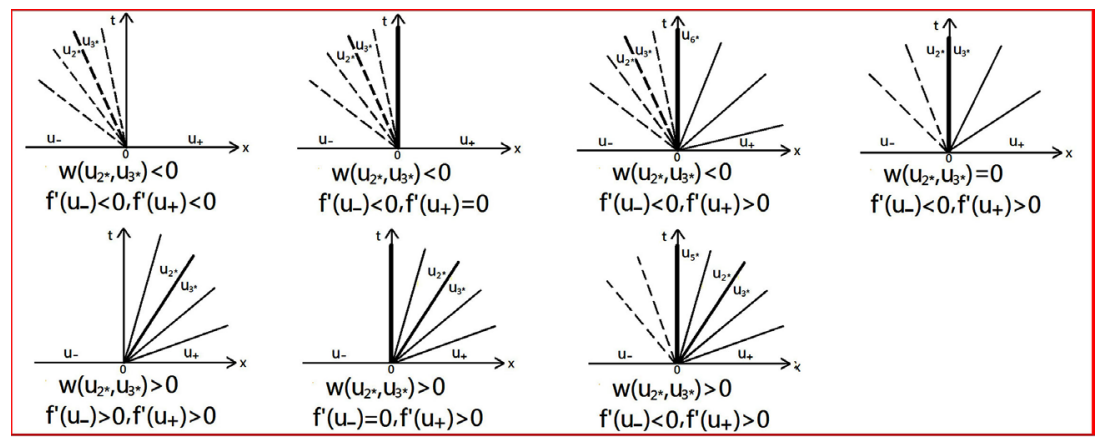

Figure 6. The expansion wave $E\left(u_{-}, u_{+}\right)$of the problem (12) for the case of $u_{+}>u_{3^{*}}$.

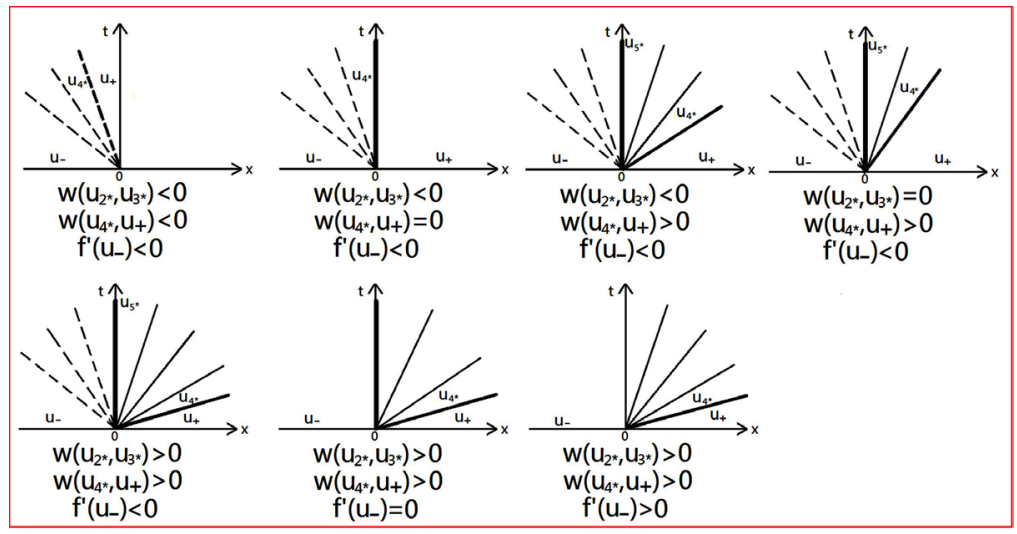

Figure 7. The expansion wave $E\left(u_{-}, u_{+}\right)$of the problem (12) for the case of $u_{d}<u_{+}<u_{3^{*}}$.

for the case of $u_{+}=u_{3^{*}}$ (see also Figure 5); and

$$
\bar{E}=\left\{\begin{aligned}
& u_{+}, \text {if } f^{\prime}\left(u_{+}\right) \leq 0 \\
& R\left(u_{6^{*}}, u_{+} ;(0,0)\right), \text { if } f^{\prime}\left(u_{+}\right)>0 \text { and } \omega\left(u_{2^{*}}, u_{3^{*}}\right) \leq 0 \\
& R\left(u_{-}, u_{2^{*}} ;(0,0)\right) \cup S^{l r}\left(u_{2^{*}}, u_{3^{*}} ;(0,0)\right) \cup R\left(u_{3^{*}}, u_{+} ;(0,0)\right), \text { if } f^{\prime}\left(u_{+}\right)>0 \text { and } \omega\left(u_{2^{*}}, u_{3^{*}}\right)>0 \text { and } f^{\prime}\left(u_{-}\right) \geq 0 \\
& R\left(u_{5^{*}}, u_{2^{*}} ;(0,0)\right) \cup S^{l r}\left(u_{2^{*}}, u_{3^{*}} ;(0,0)\right) \cup R\left(u_{3^{*}}, u_{+} ;(0,0)\right), \\
& \text { if } f^{\prime}\left(u_{+}\right)>0 \text { and } \omega\left(u_{2^{*}}, u_{3^{*}}\right)>0 \text { and } f^{\prime}\left(u_{-}\right)<0
\end{aligned}\right.
$$

for the case of $u_{+}>u_{3^{*}}$ (see also Figure 6); and

$$
\bar{E}=\left\{\begin{array}{l}
u_{+}, \text {if } \omega\left(u_{4^{*}}, u_{+}\right) \leq 0 \\
R\left(u_{-}, u_{4^{*}} ;(0,0)\right) \cup S^{l}\left(u_{4^{*}}, u_{+} ;(0,0)\right), \quad \text { if } \omega\left(u_{4^{*}}, u_{+}\right)>0 \text { and } f^{\prime}\left(u_{-}\right) \geq 0 \\
R\left(u_{5^{*}}, u_{4^{*}} ;(0,0)\right) \cup S^{l}\left(u_{4^{*}}, u_{+} ;(0,0)\right), \text { if } \omega\left(u_{4^{*}}, u_{+}\right)>0 \text { and } f^{\prime}\left(u_{-}\right)<0
\end{array}\right.
$$

for the case of $u_{d}<u_{+}<u_{3^{*}}$ (see also Figure 7).

When $u_{-}=u_{2^{*}}$,

$$
E\left(u_{-}, u_{+}\right)=\left\{\begin{array}{l}
S^{l r}\left(u_{2^{*}}, u_{3^{*}} ;(0,0)\right), \text { if } u_{+}=u_{3^{*}} \\
S^{l r}\left(u_{2^{*}}, u_{3^{*}} ;(0,0)\right) \cup R\left(u_{3^{*}}, u_{+} ;(0,0)\right), \text { if } u_{+}>u_{3^{*}} \\
R\left(u_{2^{*}}, u_{4^{*}} ;(0,0)\right) \cup S^{l}\left(u_{4^{*}}, u_{+} ;(0,0)\right), \text { if } u_{d}<u_{+}<u_{3^{*}}
\end{array}\right.
$$


and this expansion wave solution of Riemann problem (12) can be written as:

$$
v(x, t)= \begin{cases}u_{-}, & x<f^{\prime}\left(u_{3^{*}}\right) t \\ u_{+}, & x>f^{\prime}\left(u_{3^{*}}\right) t\end{cases}
$$

for $u_{+}=u_{3^{*}}$, and for $u_{+}>u_{3^{*}}$,

$$
v(x, t)=\left\{\begin{array}{l}
u_{-}, \quad x<f^{\prime}\left(u_{3^{*}}\right) t \\
\left(f^{\prime}\right)^{-1}\left(\frac{x}{t}\right), \quad f^{\prime}\left(u_{3^{*}}\right) t<x \leq f^{\prime}\left(u_{+}\right) t \\
u_{+}, \quad x>f^{\prime}\left(u_{+}\right) t
\end{array}\right.
$$

and for $u_{d}<u_{+}<u_{3^{*}}$

$$
v(x, t)=\left\{\begin{array}{l}
u_{-}, \quad x \leq f^{\prime}\left(u_{-}\right) t \\
\left(f^{\prime}\right)^{-1}\left(\frac{x}{t}\right), \quad f^{\prime}\left(u_{-}\right) t<x<f^{\prime}\left(u_{4^{*}}\right) t \\
u_{+}, \quad x>f^{\prime}\left(u_{4^{*}}\right) t
\end{array}\right.
$$

When $u_{2^{*}}<u_{-}<u_{d}$,

$$
E\left(u_{-}, u_{+}\right)=\left\{\begin{array}{l}
S^{r}\left(u_{-}, u_{7^{*}} ;(0,0)\right), \text { if } u_{+}=u_{7^{*}} \\
S^{r}\left(u_{-}, u_{7^{*}} ;(0,0)\right) \cup R\left(u_{7^{*}}, u_{+} ;(0,0)\right), \text { if } u_{+}>u_{7^{*}} \\
S\left(u_{-}, u_{+} ;(0,0)\right), \text { if } u_{d}<u_{+}<u_{7^{*}},
\end{array}\right.
$$

where $u_{7^{*}} \in\left(u_{d}, u_{3^{*}}\right)$ satisfies $\omega\left(u_{-}, u_{7^{*}}\right)=f^{\prime}\left(u_{7^{*}}\right)$. The weak entropy solution $v(x, t)$ of Riemann problem (12) $v(x, t)$ can be expressed as follows:

$$
v(x, t)= \begin{cases}u_{-}, & x<f^{\prime}\left(u_{7^{*}}\right) t \\ u_{+}, & x>f^{\prime}\left(u_{7^{*}}\right) t\end{cases}
$$

for $u_{+}=u_{7^{*}}$, and for $u_{+}>u_{7^{*}}$,

$$
v(x, t)=\left\{\begin{array}{l}
u_{-}, \quad x<f^{\prime}\left(u_{7^{*}}\right) t \\
\left(f^{\prime}\right)^{-1}\left(\frac{x}{t}\right), \quad f^{\prime}\left(u_{7^{*}}\right) t<x \leq f^{\prime}\left(u_{+}\right) t \\
u_{+}, \quad x>f^{\prime}\left(u_{+}\right) t,
\end{array}\right.
$$

and for $u_{d}<u_{+}<u_{7^{*}}$,

$$
v(x, t)= \begin{cases}u_{-}, & x<\omega\left(u_{-}, u_{+}\right) t \\ u_{+}, & x>\omega\left(u_{-}, u_{+}\right) t\end{cases}
$$

When $u_{-}=u_{2^{*}}$ or $u_{2^{*}}<u_{-}<u_{d}$, if we set $u(x, t)=\left.v(x, t)\right|_{x, t>0}$, then similar to the case of $u_{-}<u_{2^{*}}$, we can give the expression for $u(0+, t)$, and by which and Lemma 2 , it is easy to be verified that $u(x, t)$ is the global weak entropy solution of the problem (2)-(4).

\subsection{Case (II): $u_{m}=u_{-} \neq u_{+}$}

Similar to sub-section 3.1, we divide this case into two sub-cases: 1) $\left.u_{+}<u_{d}<u_{-} ; 2\right) \quad u_{-}<u_{d}<u_{+}$. 
3.2.1. $u_{+}<u_{d}<u_{-}$

Consider the following initial value problem

$$
\left\{\begin{array}{l}
v_{t}+f(v)_{x}=0,-\infty<x<\infty, t>0 \\
v(x, 0)= \begin{cases}u_{-}, & x<a \\
u_{+}, & x>a\end{cases}
\end{array}\right.
$$

Let $u_{i^{*}}(i=1,2, \cdots, 7)$ be defined in sub-section 3.1.

If $u_{+} \leq u_{1^{*}}$, the weak entropy solution $v(x, t)$ of Riemann problem (35) includes only a shock wave $S\left(u_{-}, u_{+} ;(a, 0)\right)$ starting at point $(a, 0)$, and this shock wave solution can be expressed as follows:

$$
v(x, t)= \begin{cases}u_{-}, & x<\omega\left(u_{-}, u_{+}\right) t \\ u_{+}, & x>\omega\left(u_{-}, u_{+}\right) t\end{cases}
$$

Let $u(x, t)=\left.v(x, t)\right|_{x, t>0}$, then as $\omega\left(u_{-}, u_{+}\right) \geq 0, u(0+, t)=u_{-} \quad(t>0)$, and as $\omega\left(u_{-}, u_{+}\right)<0$,

$$
u(0+, t)= \begin{cases}u_{-}, & 0<t<-a\left(\omega\left(u_{-}, u_{+}\right)\right)^{-1} \\ u_{+}, & t>-a\left(\omega\left(u_{-}, u_{+}\right)\right)^{-1}\end{cases}
$$

where $t=-a\left(\omega\left(u_{-}, u_{+}\right)\right)^{-1}$ is the intersection time of $S\left(u_{-}, u_{+} ;(a, 0)\right)$ and $t$-axis. Thus by Lemma 2 , we can derive that $u(x, t)$ is the global weak entropy solution of the initial-boundary problem (2)-(4). $u(x, t)$ includes only a shock wave $S\left(u_{-}, u_{+} ;(a, 0)\right)$, which will be far away from the boundary $x=0$ as $\omega\left(u_{-}, u_{+}\right) \geq 0$ or will interact with the boundary and be absorbed by the boundary at time $t=t_{1}$ (see Figure 8).

If $u_{1^{*}}<u_{+}<u_{d}$, a compression wave $C\left(u_{-}, u_{+}\right)$, which includes two shock waves $S\left(u_{-}, u_{d} ;(a, 0)\right)$ and $S\left(u_{d}, u_{+} ;(a, 0)\right)$, i.e., $C\left(u_{-}, u_{+}\right)=S\left(u_{-}, u_{d} ;(a, 0)\right) \cup S\left(u_{d}, u_{+} ;(a, 0)\right)$, appears in the weak entropy solution $v(x, t)$ of the Riemann problem (35), this compression wave solution $v(x, t)$ can be written as:

$$
v(x, t)= \begin{cases}u_{-}, & x<a+\omega\left(u_{-}, u_{d}\right) t \\ u_{d}, & a+\omega\left(u_{-}, u_{d}\right) t<x<a+\omega\left(u_{d}, u_{+}\right) t \\ u_{+}, & x>a+\omega\left(u_{d}, u_{+}\right) t\end{cases}
$$

We take $u(x, t)=\left.v(x, t)\right|_{x, t>0}$, then the expression for $u(0+, t)$ can be given as follows. As $\omega\left(u_{-}, u_{d}\right) \geq 0, u(0+, t)=u_{-} \quad(t>0)$; as $\omega\left(u_{-}, u_{d}\right)<0$ and $\omega\left(u_{d}, u_{+}\right) \geq 0$,

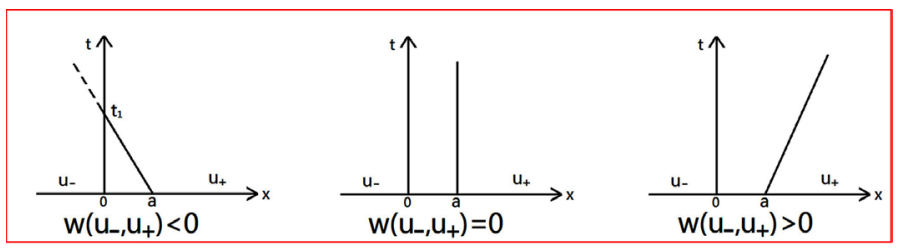

Figure 8. The interaction of the shock wave $S\left(u_{-}, u_{+} ;(a, 0)\right)$ with the boundary $x=0$. 


$$
u(0+, t)= \begin{cases}u_{-}, & 0<t<-a / \omega\left(u_{-}, u_{d}\right) \\ u_{d}, & t>-a / \omega\left(u_{-}, u_{d}\right)\end{cases}
$$

as $\omega\left(u_{d}, u_{+}\right)<0$,

$$
u(0+, t)= \begin{cases}u_{-}, & 0<t<-a / \omega\left(u_{-}, u_{d}\right) \\ u_{d}, & -a / \omega\left(u_{-}, u_{d}\right)<t<-a / \omega\left(u_{d}, u_{+}\right) \\ u_{+}, & t>-a / \omega\left(u_{d}, u_{+}\right)\end{cases}
$$

Therefore, from Lemma 2, it follows that $u(x, t)$ is the global weak entropy solution of the initial-boundary problem (2)-(4). $u(x, t)$ includes only a compression wave $\left.C\left(u_{-}, u_{+}\right)\right|_{x, t>0}$, which will be far away from the boundary $x=0$ (if $\left.\omega\left(u_{-}, u_{d}\right) \geq 0\right)$ or will interact with the boundary and be partially absorbed (if $\omega\left(u_{-}, u_{d}\right)<0$ and $\left.\omega\left(u_{d}, u_{+}\right) \geq 0\right)$ or completely absorbed (if $\left.\omega\left(u_{d}, u_{+}\right)<0\right)$ by the boundary (see Figure 9).

\subsection{2. $u_{-}<u_{d}<u_{+}$}

The weak entropy solution $v(x, t)$ of Riemann problem (35) also includes only an expansion wave $E\left(u_{-}, u_{+}\right)$.

When $u_{-}<u_{2^{*}}, E\left(u_{-}, u_{+}\right)$can be expressed as follows:

$$
E\left(u_{-}, u_{+}\right)=\left\{\begin{array}{l}
R\left(u_{-}, u_{2^{*}} ;(a, 0)\right) \cup S^{l r}\left(u_{2^{*}}, u_{3^{*}} ;(a, 0)\right), \text { if } u_{+}=u_{3^{*}} \\
R\left(u_{-}, u_{2^{*}} ;(a, 0)\right) \cup S^{l r}\left(u_{2^{*}}, u_{3^{*}} ;(a, 0)\right) \cup R\left(u_{3^{*}}, u_{+} ;(a, 0)\right), \text { if } u_{+}>u_{3^{*}} \\
R\left(u_{-}, u_{4^{*}} ;(a, 0)\right) \cup S^{l}\left(u_{4^{*}}, u_{+} ;(a, 0)\right), \text { if } u_{d}<u_{+}<u_{3^{*}}
\end{array}\right.
$$

Thus this expansion wave solution can be written as:

$$
v(x, t)=\left\{\begin{array}{l}
u_{-}, \quad x \leq a+f^{\prime}\left(u_{-}\right) t \\
\left(f^{\prime}\right)^{-1}\left(\frac{x-a}{t}\right), \quad a+f^{\prime}\left(u_{-}\right) t<x<a+f^{\prime}\left(u_{2^{*}}\right) t \\
u_{+}, \quad x>a+f^{\prime}\left(u_{2^{*}}\right) t
\end{array}\right.
$$

for $u_{+}=u_{3^{*}}$, and for $u_{+}>u_{3^{*}}$,

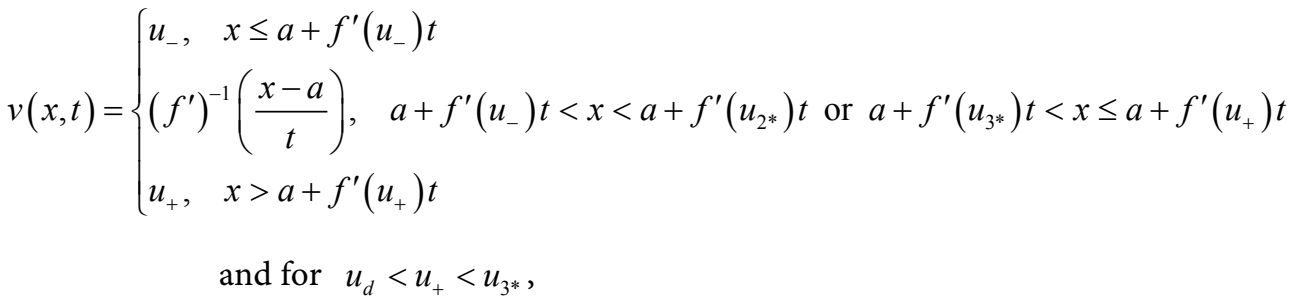

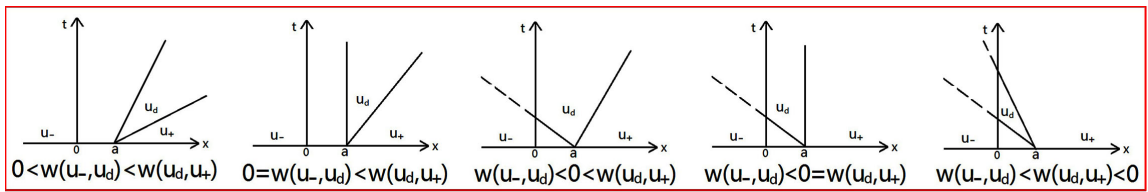

Figure 9. The interaction of the compression wave $\left.C\left(u_{-}, u_{+}\right)\right|_{x, t>0}$ with the boundary $x=0$. 


$$
v(x, t)=\left\{\begin{array}{l}
u_{-}, \quad x \leq a+f^{\prime}\left(u_{-}\right) t \\
\left(f^{\prime}\right)^{-1}\left(\frac{x-a}{t}\right), \quad a+f^{\prime}\left(u_{-}\right) t<x<a+f^{\prime}\left(u_{4^{*}}\right) t \\
u_{+}, \quad x>a+f^{\prime}\left(u_{4^{*}}\right) t
\end{array}\right.
$$

When $u_{-}=u_{2^{*}}$,

$$
E\left(u_{-}, u_{+}\right)=\left\{\begin{array}{l}
S^{l r}\left(u_{2^{*}}, u_{3^{*}} ;(a, 0)\right), \text { if } u_{+}=u_{3^{*}} \\
S^{l r}\left(u_{2^{*}}, u_{3^{*}} ;(a, 0)\right) \cup R\left(u_{3^{*}}, u_{+} ;(a, 0)\right), \text { if } u_{+}>u_{3^{*}} \\
R\left(u_{2^{*}}, u_{4^{*}} ;(a, 0)\right) \cup S^{l}\left(u_{4^{*}}, u_{+} ;(a, 0)\right), \text { if } u_{d}<u_{+}<u_{3^{*}}
\end{array}\right.
$$

This expansion wave solution can be written as:

$$
v(x, t)= \begin{cases}u_{-}, & x<a+f^{\prime}\left(u_{3^{*}}\right) t \\ u_{+}, & x>a+f^{\prime}\left(u_{3^{*}}\right) t\end{cases}
$$

for $u_{+}=u_{3^{*}}$, and for $u_{+}>u_{3^{*}}$,

$$
v(x, t)=\left\{\begin{array}{l}
u_{-}, \quad x<a+f^{\prime}\left(u_{3^{*}}\right) t \\
\left(f^{\prime}\right)^{-1}\left(\frac{x-a}{t}\right), \quad a+f^{\prime}\left(u_{3^{*}}\right) t<x \leq a+f^{\prime}\left(u_{+}\right) t \\
u_{+}, \quad x>a+f^{\prime}\left(u_{+}\right) t
\end{array}\right.
$$

and for $u_{d}<u_{+}<u_{3^{*}}$,

$$
v(x, t)=\left\{\begin{array}{l}
u_{-}, \quad x \leq a+f^{\prime}\left(u_{-}\right) t \\
\left(f^{\prime}\right)^{-1}\left(\frac{x-a}{t}\right), \quad a+f^{\prime}\left(u_{-}\right) t<x<a+f^{\prime}\left(u_{4^{*}}\right) t \\
u_{+}, \quad x>a+f^{\prime}\left(u_{4^{*}}\right) t
\end{array}\right.
$$

When $u_{2^{*}}<u_{-}<u_{d}$,

$$
E\left(u_{-}, u_{+}\right)=\left\{\begin{array}{l}
S^{r}\left(u_{-}, u_{7^{*}} ;(a, 0)\right), \text { if } u_{+}=u_{7^{*}} \\
S^{r}\left(u_{-}, u_{7^{*}} ;(a, 0)\right) \cup R\left(u_{7^{*}}, u_{+} ;(a, 0)\right), \text { if } u_{+}>u_{7^{*}} \\
S\left(u_{-}, u_{+} ;(a, 0)\right), \text { if } u_{d}<u_{+}<u_{7^{*}},
\end{array}\right.
$$

The weak entropy solution $v(x, t)$ of Riemann problem (35) $v(x, t)$ can be expressed as follows:

$$
v(x, t)= \begin{cases}u_{-}, & x<a+f^{\prime}\left(u_{7^{*}}\right) t \\ u_{+}, & x>a+f^{\prime}\left(u_{7^{*}}\right) t\end{cases}
$$

for $u_{+}=u_{7^{*}}$, and for $u_{+}>u_{7^{*}}$,

$$
v(x, t)=\left\{\begin{array}{l}
u_{-}, \quad x<a+f^{\prime}\left(u_{7^{*}}\right) t \\
\left(f^{\prime}\right)^{-1}\left(\frac{x-a}{t}\right), \quad a+f^{\prime}\left(u_{7^{*}}\right) t<x \leq a+f^{\prime}\left(u_{+}\right) t \\
u_{+}, \quad x>a+f^{\prime}\left(u_{+}\right) t
\end{array}\right.
$$

and for $u_{d}<u_{+}<u_{7^{*}}$,

$$
v(x, t)= \begin{cases}u_{-}, & x<a+\omega\left(u_{-}, u_{+}\right) t \\ u_{+}, & x>a+\omega\left(u_{-}, u_{+}\right) t\end{cases}
$$


Let $u(x, t)=\left.v(x, t)\right|_{x, t>0}$, then by using of Lemma 2 and the expression of $v(x, t)$, we can also verify that $u(x, t)$ is the global weak entropy solution of the problem (2)-(4). In what follows, we write the expression for $u(0+, t)$ and state the interaction of the elementary wave and the boundary only for the case of $u_{-}<u_{2^{*}}$, and we can deal with the other cases similarly.

If $u_{-}<u_{2^{*}}$ and $u_{+}=u_{3^{*}}$, then $u(0+, t)=u_{-}(t>0)$ as $f^{\prime}\left(u_{-}\right) \geq 0$, and

$$
u(0+, t)=\left\{\begin{array}{l}
u_{-}, 0<t \leq-a / f^{\prime}\left(u_{-}\right) \\
\left(f^{\prime}\right)^{-1}\left(\frac{x-a}{t}\right), t>-a / f^{\prime}\left(u_{-}\right)
\end{array}\right.
$$

as $f^{\prime}\left(u_{-}\right)<0$ and $f^{\prime}\left(u_{2^{*}}\right) \geq 0$, and

$$
u(0+, t)=\left\{\begin{array}{l}
u_{-}, 0<t \leq-a / f^{\prime}\left(u_{-}\right) \\
\left(f^{\prime}\right)^{-1}\left(\frac{x-a}{t}\right),-a / f^{\prime}\left(u_{-}\right)<t<-a / f^{\prime}\left(u_{+}\right) \\
u_{+}, t>-a / f^{\prime}\left(u_{+}\right)
\end{array}\right.
$$

as $f^{\prime}\left(u_{2^{*}}\right)<0$. In this case, $u(x, t)$ includes only a expansion wave $\left.E\left(u_{-}, u_{+}\right)\right|_{x, t>0}$. It will be far away from the boundary as $f^{\prime}\left(u_{-}\right) \geq 0$, or one part $R\left(u_{-}, u_{5^{*}} ;(a, 0)\right)$ of rarefaction wave in $\left.E\left(u_{-}, u_{+}\right)\right|_{x, t>0}$ will be absorbed by the boundary as $f^{\prime}\left(u_{-}\right)<0$ and $f^{\prime}\left(u_{2^{*}}\right)>0$, or the whole of $\left.E\left(u_{-}, u_{+}\right)\right|_{x, t>0}$ will be completely absorbed by the boundary as $f^{\prime}\left(u_{2^{*}}\right) \leq 0 \quad$ (see Figure 10).

If $u_{-}<u_{2^{*}}$ and $u_{+}>u_{3^{*}}$, then $u(0+, t)=u_{-}(t>0)$ as $f^{\prime}\left(u_{-}\right) \geq 0$, and

$$
u(0+, t)=\left\{\begin{array}{l}
u_{-}, 0<t \leq-a / f^{\prime}\left(u_{-}\right) \\
\left(f^{\prime}\right)^{-1}\left(\frac{x-a}{t}\right), t>-a / f^{\prime}\left(u_{-}\right)
\end{array}\right.
$$

as $f^{\prime}\left(u_{-}\right)<0$ and $f^{\prime}\left(u_{2^{*}}\right) \geq 0$, and

$$
u(0+, t)=\left\{\begin{array}{l}
u_{-}, 0<t \leq-a / f^{\prime}\left(u_{-}\right) \\
\left(f^{\prime}\right)^{-1}\left(\frac{x-a}{t}\right), t>-a / f^{\prime}\left(u_{-}\right), t \neq-a / \omega\left(u_{2^{*}}, u_{3^{*}}\right)
\end{array}\right.
$$

as $f^{\prime}\left(u_{2^{*}}\right)<0$ and $f^{\prime}\left(u_{+}\right) \geq 0$, and

$$
u(0+, t)=\left\{\begin{array}{l}
u_{-}, 0<t \leq-a / f^{\prime}\left(u_{-}\right) \\
\left(f^{\prime}\right)^{-1}\left(\frac{x-a}{t}\right),-a / f^{\prime}\left(u_{-}\right)<t \leq-a / f^{\prime}\left(u_{+}\right), t \neq-a / \omega\left(u_{2^{*}}, u_{3^{*}}\right) \\
u_{+}, t>-a / f^{\prime}\left(u_{+}\right)
\end{array}\right.
$$

as $f^{\prime}\left(u_{+}\right)<0$. In this case, $u(x, t)$ includes only a expansion wave $\left.E\left(u_{-}, u_{+}\right)\right|_{x, t>0}$. It will be far away from the boundary as $f^{\prime}\left(u_{-}\right) \geq 0$, or one part $R\left(u_{-}, u_{5^{*}} ;(a, 0)\right)$ of rarefaction wave in $\left.E\left(u_{-}, u_{+}\right)\right|_{x, t>0}$ will be absorbed by the boundary as $f^{\prime}\left(u_{-}\right)<0$ and $f^{\prime}\left(u_{2^{*}}\right) \geq 0$, or one part

$R\left(u_{-}, u_{2^{*}} ;(a, 0)\right) \cup S^{l r}\left(u_{2^{*}}, u_{3^{*}} ;(a, 0)\right) \cup R\left(u_{3^{*}}, u_{6^{*}} ;(a, 0)\right)$ of $\left.E\left(u_{-}, u_{+}\right)\right|_{x, t>0} \quad$ will be absorbed by the boundary as $f^{\prime}\left(u_{2^{*}}\right)<0$ and $f^{\prime}\left(u_{+}\right)>0$, or the whole of $\left.E\left(u_{-}, u_{+}\right)\right|_{x, t>0}$ will be completely absorbed by the boundary $f^{\prime}\left(u_{+}\right) \leq 0 \quad$ (see Figure 11). 
If $u_{-}<u_{2^{*}}$ and $u_{d}<u_{+}<u_{3^{*}}$, then $u(0+, t)=u_{-}(t>0)$ as $f^{\prime}\left(u_{-}\right) \geq 0$, and

$$
u(0+, t)=\left\{\begin{array}{l}
u_{-}, 0<t \leq-a / f^{\prime}\left(u_{-}\right) \\
\left(f^{\prime}\right)^{-1}\left(\frac{x-a}{t}\right), t>-a / f^{\prime}\left(u_{-}\right)
\end{array}\right.
$$

as $f^{\prime}\left(u_{-}\right)<0$ and $f^{\prime}\left(u_{4^{*}}\right) \geq 0$, and

$$
u(0+, t)=\left\{\begin{array}{l}
u_{-}, 0<t \leq-a / f^{\prime}\left(u_{-}\right) \\
\left(f^{\prime}\right)^{-1}\left(\frac{x-a}{t}\right),-a / f^{\prime}\left(u_{-}\right)<t<-a / f^{\prime}\left(u_{4^{*}}\right) \\
u_{+}, t>-a / f^{\prime}\left(u_{4^{*}}\right)
\end{array}\right.
$$

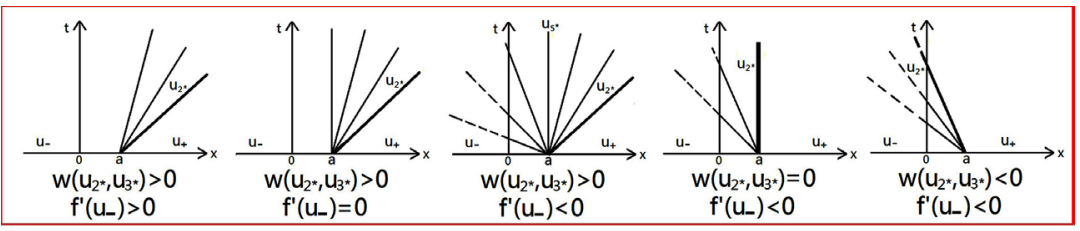

Figure 10. The interaction of the expansion wave $\left.E\left(u_{-}, u_{+}\right)\right|_{x, t>0}$ with the boundary $x=0$ for the case of $u_{-}<u_{2^{*}}$ and $u_{+}=u_{3^{*}}$.

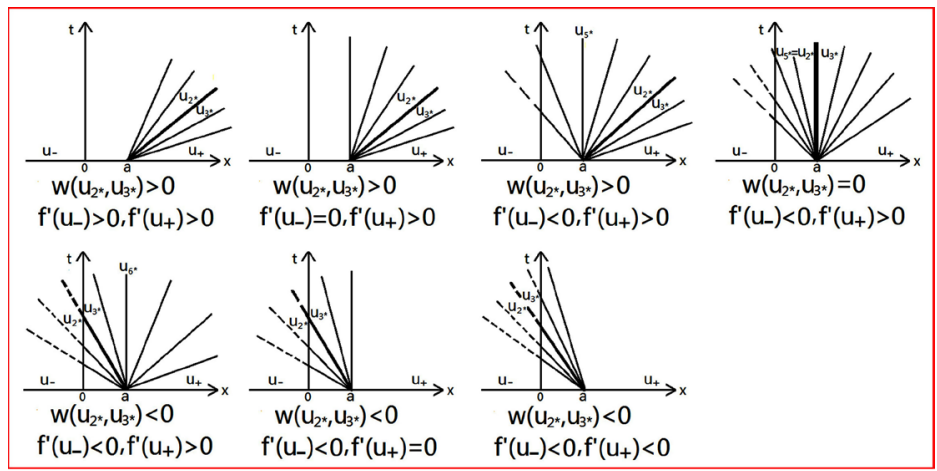

Figure 11. The interaction of the expansion wave $\left.E\left(u_{-}, u_{+}\right)\right|_{x, t>0}$ with the boundary $x=0$ for the case of $u_{-}<u_{2^{*}}$ and $u_{+}>u_{3^{*}}$.

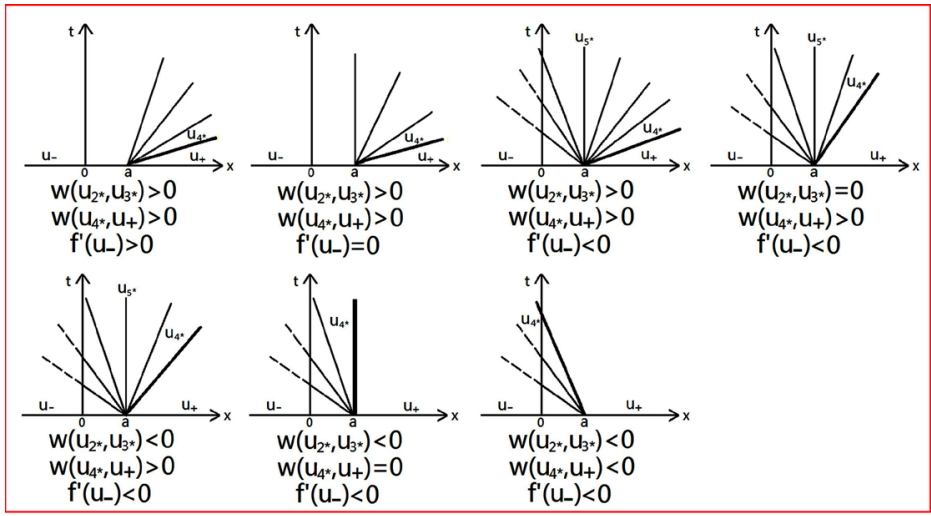

Figure 12. The interaction of the expansion wave $\left.E\left(u_{-}, u_{+}\right)\right|_{x, t>0}$ with the boundary $x=0$ for the case of $u_{-}<u_{2^{*}}$ and $u_{d}<u_{+}<u_{3^{*}}$. 
as $f^{\prime}\left(u_{4^{*}}\right)<0$. We now state the interaction of the unique elementary wave $\left.E\left(u_{-}, u_{+}\right)\right|_{x, t>0}$ in $u(x, t)$ with the boundary for this case. $\left.E\left(u_{-}, u_{+}\right)\right|_{x, t>0}$ will be far away from the boundary as $f^{\prime}\left(u_{-}\right) \geq 0$, or one part $R\left(u_{-}, u_{5^{*}} ;(a, 0)\right)$ of rarefaction wave in $\left.E\left(u_{-}, u_{+}\right)\right|_{x, t>0}$ will be absorbed by the boundary as $f^{\prime}\left(u_{-}\right)<0$ and $f^{\prime}\left(u_{4^{*}}\right)>0$ or the whole of $\left.E\left(u_{-}, u_{+}\right)\right|_{x, t>0}$ will be completely absorbed by the boundary $f^{\prime}\left(u_{4^{*}}\right) \leq 0$ (see Figure 12).

\section{Conclusion}

The main purpose of our present manuscript is devoted to studying the structure of the global weak entropy solution for the above initial-boundary value problem under the condition of $f_{-}^{\prime}\left(u_{d}\right)>f_{+}^{\prime}\left(u_{d}\right)$. By the characteristic method and the truncation method, we construct the global weak entropy solution of this initial-boundary value problem, and investigate the interaction of elementary waves with the boundary and the boundary behavior of the weak entropy solution. Compared with the case of $f_{-}^{\prime}\left(u_{d}\right)<f_{+}^{\prime}\left(u_{d}\right)$, the weak entropy solution of the problem (2)-(4) with the case of $f_{-}^{\prime}\left(u_{d}\right)>f_{+}^{\prime}\left(u_{d}\right)$ includes different wave type: contact discontinuity.

\section{Acknowledgements}

This work was supported by the National Natural Science Foundation of China (No. 11731008) and the Guangdong Natural Science Foundation of China (2018A030313906).

\section{Conflicts of Interest}

The authors declare no conflicts of interest regarding the publication of this paper.

\section{References}

[1] Dai, Y.H. and Zhang, J. (2017) Construction of Global Weak Entropy Solution of Initial-Boundary Value Problem for Scalar Conservation Laws with Weak Discontinuity Flux. American Journal of Computational Mathematics, 7, 451-468. https://doi.org/10.4236/ajcm.2017.74033

[2] Bardos, C., Leroux, A.Y. and Nedelec, J.C. (1979) First Order Quasilinear Equations with Boundary Conditions. Communications in Partial Differential Equations, 4, 1017-1034. https://doi.org/10.1080/03605307908820117

[3] Pan, T. and Lin, L.W. (1995) The Global Solution of the Scalar Nonconvex Conservation Laws with Boundary Condition. Journal of Partial Differential Equations, 8, 371-383.

[4] Szepessy, A. (1989) Measure-Value Solution to Scalar Conservation Laws with Boundary Conditions. Archive for Rational Mechanics and Analysis, 139, 181-193. https://doi.org/10.1007/BF00286499

[5] Joseph, K.T. (1988) Burgers Equation in the Quarter Plane: A Formula for the Weak Limit. Communications on Pure and Applied Mathematics, 41, 133-149. https://doi.org/10.1002/cpa.3160410202

[6] Dubotis, F. and LeFloch, P.G. (1988) Boundary Conditions for Nonlinear Hyper- 
bolic System of Conservation Laws. Journal of Differential Equations, 8, 93-122. https://doi.org/10.1016/0022-0396(88)90040-X

[7] Chen, G.Q. and Frid, H. (2000) Vanishing Viscosity Limit for Initial-Boundary Value Problems for Conservation Laws. American Mathematical Society, 238, 35-51.

[8] Pan, T. and Lin, L.W. (1998) The Global Solution of the Scalar Nonconvex Conservation Laws with Boundary Condition (Continuation). Journal of Partial Differential Equations, 11, 1-8.

[9] LeFloch, P.G. (1988) Explicit Formula for Nonlinear Conservation Laws with Boundary Conditions. Mathematical Methods in the Applied Sciences, 10, 265-287. https://doi.org/10.1002/mma.1670100305

[10] Bustos, M.C., Concha, F. and Wendland, W.L. (1990) Global Weak Solution to the Problem of Continuous Sedimentation of an Ideal Suspension. Mathematical Methods in the Applied Sciences, 13, 1-22. https://doi.org/10.1002/mma.1670130102

[11] Bustos, M.C., Paiva, F. and Wendland, W.L. (1996) Entropy Boundary Condition in the Theory of Sedimentation of Ideal Suspension. Mathematical Methods in the Applied Sciences, 19, 679-697. https://doi.org/10.1002/(SICI)1099-1476(199606)19:9\%3C679::AID-MMA784\%3E3. $\underline{0 . \mathrm{CO} ; 2-\mathrm{L}}$

[12] Bustos, M.C. and Concha, F. (1988) On the Construction of Global Weak Solutions in the Kynch Theory of Sedimentation. Mathematical Methods in the Applied Sciences, 10, 245-264. https://doi.org/10.1002/mma.1670100304

[13] Liu, H.X. and Pan, T. (2004) L1-Convergence Rate of Viscosity Methods for Scalar Conservation Laws with the Interaction of Elementary Waves and the Boundary. Quarterly of Applied Mathematics, 4, 601-621. https://doi.org/10.1090/qam/2104264

[14] Liu, H.X. and Pan, T. (2007) Construction of Solutions and L1-Error Estimates of Viscous Methods for Scalar Conservation Laws with Boundary. Acta Mathematica Sinica, 23, 393-410.

[15] Liu, H.X. and Pan, T. (2003) Interaction of Elementary Waves for Scalar Conservation Laws on a Bounded Domain. Mathematical Methods in the Applied Sciences, 26, 619-632. https://doi.org/10.1002/mma.370

[16] Hopf, E. (1969) On the Right Weak Solution of the Cauchy Problem for a Quasilinear Equation of First Order. Journal of Mathematics and Mechanics, 19, 483-487.

[17] Zhang, T. and Hsiao, L. (1989) The Riemann Problem and Interaction of Waves in Gas Dynamics. In: Pitman Monographs and Surveys in Pure and Applied Mathematics, Vol. 41, Longman Sci. Techn., Harlow. 